\title{
DESCRIÇÃO DE GENITÁLIAS DE Neoblattella SHELFORD, 1911 COM CHAVE PARA IDENTIFICAÇÃO E CONSIDERAÇÕES SOBRE O GÊNERO (BLATTARIA, BLATTELLIDAE)
}

\section{Sonia Maria Lopes \& Edivar Heeren de Oliveira}

\begin{abstract}
Description of genitalia of Neoblattella Shelford, 1911 with key for identification and considerations about the genus (Blattaria, Blattellidae) - In this paper seven species of Neoblattella Shelford, 1911 are studied and, in first time, described the female and male's genitalia. The species are gathered in four groups with the purpose of establishing relationship among them and to elucidate taxonomics problems.
\end{abstract}

Key words: Neoblattella, Blattaria, Blattellidae, genitalia, taxonomy

\section{Resumo}

Neste trabalho sete espécies de Neoblattella Shelford, 1911 são estudadas e descritas, pela primeira vez, as genitálias do macho e da fêmea. Com base nesse estudo são reunidas em quatro grupos, com a finalidade de estabelecer relação entre elas e elucidar problemas taxonômicos.

Palavras-chave: Neoblattella, Blattaria, Blattellidae, genitália, taxonomia

Departamento de Entomologia, Museu Nacional, Universidade Federal do Rio de Janeiro, Quinta da Boa Vista, CEP: 20940-040 São Cristóvão, Rio de Janeiro, Brasil. E-mail: sonialf@acd.ufrj.br

Recebido em: 01/08/2003.

Aceito em: 22/11/2004. 


\section{Introdução}

As espécies de Neoblattella caracterizam-se por apresentar no macho: 1) abdome com modificação tergal no sexto, sétimo e oitavo segmentos ou somente no sétimo segmento, em forma de cílios dispersos; 2) placa supra-anal prolongada entre os cercos com as margens laterais retas e convergentes; 3 ) placa subgenital simétrica, estilos iguais afilados dispostos próximos aos bordos da placa; 4) L2vm bifurcado com L2d de formas diversas.

O gênero conta com 20 espécies, das quais sete foram estudadas neste trabalho, em razão da disponibilidade existente na coleção do Museu Nacional. Com esse material foi feito um estudo pioneiro das genitálias do macho e da fêmea de algumas dessas espécies, visando estabelecer a relação entre elas, para elucidar problemas taxonômicos. As espécies foram reunidas em complexos, e novos registros no Brasil foram acrescentados.

As espécies de Neoblattella com distribuição na região neotropical desde América Central até o sul da América do Sul (Fig. 55) são de habitat preferencialmente bromelícola, podendo ser encontradas também na vegetação e em folhiço dentro da mata.

O gênero Neoblattella foi descrito por Shelford (1911) com base em Blatta adspersicollis Stal, 1858.

Rehn (1915) a partir de uma fêmea descreveu uma espécie nova da Argentina (N. puerilis) e em 1932 uma espécie nova do Estado do Pará (N. sucina).

Bruijning (1959) estudando o gênero Neoblattella considerou à ele pertencentes somente seis espécies, englobando-as no grupo que denominou como adspersicollis ( $N$. adspersicollis (Stal, 1861); $N$. binodosa Hebard, 1926; N. poecilops Hebard, 1926; N. longior Hebard, 1926; N. unifascia Hebard, 1926 e N. guianae Hebard, 1929).

\section{Material e Métodos}

Os espécimes foram analisados morfologicamente de acordo com as técnicas rotineiras desenvolvidas em laboratório e descritas em Lopes \& Oliveira (2000). No texto utilizamos asterisco $\left(^{*}\right)$ para designar novas ocorrências, e as seguintes abreviaturas: L1 para falômero esquerdo; L2d para ápice do esclerito mediano; L2vm para esclerito mediano; R2 para 
falômero direito; R3 para esclerito do falômero direito, e no catálogo o local de depósito dos tipos nas Instituições como a seguir: AMNH para American Museum of Natural History, N. York; ANSP para Academy of Natural Sciences, Philadelphia; BMNH para British Museum National History; MNHP para Museum Histoire Naturelle de France; MNRJ para Museu Nacional do Rio de Janeiro; USNM para United States National Museum.

\section{Resultados}

\section{Neoblattella Shelford, 1911}

NeoblattellaShelford, 1911:155; Caudell, 1913:603 (Blattella); Hebard, 1916:159; 1919:58; 1922a:226; 1926: 159; 1932:204; Rehn \& Hebard, 1927:54; Gurney, 1942:20; Rehn, 1949:12; Bruijning, 1959:60; Bonfils, 1969:115.

Espécie-tipo: Blatta adspersicollis Stal, 1858.

\section{N. adspersicollis (Stal, 1858)}

adspersicollis Stal, 1858-1861:308 (Blatta); Brunner, 1865:107; Saussure, 1870: 35; Walker, 1868:97 e 236; Brunner \& Redtenbacher, 1892:202; Brunner, 1893:607; Saussure \& Zehntner, 1893:42-43; Kirby, 1904:94; Bruner, 1906:139; Shelford, 1908:14; 1911:155; Gowdey, 1926:5 (Phyllodromia); Rehn \& Hebard, 1905:32; 1914:379; Caudell, 1913:603; (Neoblattella); Hebard, 1917:38 (Latiblattella); Rehn \& Hebard, 1912:239; Rehn, 1916:230; 1949:12; Hebard, 1922:198 e 232; 1926:163; 1929:357; Wolcott, 1924:19; Princis \& Kevan, 1955:167; Bruijning, 1959:60; Rocha e Silva-Albuquerque \& Gurney, 1962:247; Rocha e Silva-Albuquerque, 1964a:27; 1972:27; Rocha e Silva, 1982:14.

Localidade-tipo: Macho. BRASIL, Rio de Janeiro (ANSP).

Distribuição geográfica: BRASIL (Amapá, Pará, Ceará, Bahia, Mato Grosso, Rio de Janeiro), TRINIDAD, JAMAICA, PORTO RICO, GUIANA FRANCESA, COLÔMBIA.

\section{N. binodosa Hebard, 1926}

binodosa Hebard, 1926:165; Bruijning, 1959:61; Rocha e Silva-Albuquerque, 1964a: 27; 1972:27. 
Localidade-tipo: Macho. GUIANA FRANCESA (ST. Jean du Maroni), (ANSP). Distribuição geográfica: BRASIL (Pará, Bahia*, São Paulo), GUIANA FRANCESA E SURINAME.

\section{N. carvalhoi Rocha e Silva-Albuquerque \& Lopes, 1976}

carvalhoi Rocha e Silva-Albuquerque \& Lopes, 1976:895.

Localidade-tipo: Macho. BRASIL (Rio de Janeiro) (MNRJ).

Distribuição Geográfica: BRASIL (Rio de Janeiro).

\section{N. elegantula Rocha e Silva-Albuquerque, 1964}

elegantula Rocha e Silva-Albuquerque, 1964b:14.

Localidade-tipo: Macho. BRASIL (Amazonas) (USNM).

Distribuição Geográfica: BRASIL (Amazonas), VENEZUELA.

\section{N. guianae Hebard, 1929}

guianae Hebard, 1929:356; Bruijning, 1959:62; Rocha e Silva-Albuquerque \& Gurney, 1962:248; Rocha e Silva-Albuquerque, 1964a:27; 1964c:3; 1972:28.

Localidade-tipo: Macho. GUIANA (Kartabo) (ANSP).

Distribuição geográfica: BRASIL (Amapá, Pará, Mato Grosso*, São PauIo*), GUIANA FRANCESA, GUIANA, PERU.

\section{N. longior Hebard, 1926}

longior Hebard, 1926:167; Bruijning, 1959:62; Rocha e Silva-Albuquerque, 1965:8; 1972:28.

Localidade-tipo: Macho. GUIANA FRANCESA (St. Jean du Maroni), (ANSP). Distribuição geográfica: BRASIL (Pará, Mato Grosso*), GUIANA FRANCESA, SURINAME.

\section{N. paulista Rocha e Silva-Albuquerque \& Gurney, 1963}

paulista Rocha e Silva-Albuquerque \& Gurney, 1963:526, 527; Rocha e Silva-Albuquerque, 1964a:27. 
Localidade-tipo: Macho. BRASIL: São Paulo (USNM)

Distribuição Geográfica: BRASIL (São Paulo)

\section{N. picta Rocha e Silva-Albuquerque \& Gurney, 1962}

picta Rocha e Silva-Albuquerque \& Gurney, 1962:248; Rocha e SilvaAlbuquerque, 1964a:27; 1972:28; Rocha e Silva-Albuquerque \& Lopes, 1976:895; Rocha e Silva, 1982:14. Localidade-tipo: Macho. BRASIL (Amapá) (USNM).

Distribuição Geográfica: BRASIL (Amapá, Espírito Santo, Rio de Janeiro).

\section{N. poecilops Hebard, 1926}

poecilops Hebard, 1926:164; Bruijning, 1959:61; Rocha e Silva-Albuquerque, 1972:28.

Localidade-tipo: Macho. GUIANA FRANCESA (St. Jean du Maroni), (ANSP). Distribuição Geográfica: BRASIL (Pará, Mato Grosso, Pernambuco*), GUIANA FRANCESA, SURINAME.

\section{N. puerilis Rehn, 1915}

puerilis Rehn, 1915:273 (Ceratinoptera); Hebard, 1922: 262; Becker, 1979:19-21.

Localidade-tipo: Macho.ARGENTINA (Missiones) (ANSP).

Distribuição Geográfica: BRASIL (Rio Grande do Sul), ARGENTINA.

\section{N. sucina Rehn, 1932}

sucina Rehn, 1932:24; Rocha e Silva-Albuquerque, 1964a: 27.

Localidade-tipo: Macho. BRASIL (Pará) (ANSP)

Distribuição Geográfica: BRASIL (Amazonas, Pará).

\section{N. tapenagae Hebard, 1922}

tapenagae Hebard, 1922:262-264.

Localidade-tipo: Fêmea. ARGENTINA (Chaco) (MNHP)

Distribuição Geográfica: ARGENTINA 


\section{N. titania (Rehn, 1903)}

titania Rehn, 1903:267 (Blattella); Hebard, 1926:160; Rehn, 1932:24; Bruijning, 1959:66; Rocha e Silva-Albuquerque, 1964a:27; 1972:28. Localidade-tipo: Macho. GUIANA (Bartica) (ANSP).

Distribuição Geográfica: BRASIL (Amazonas, Pará), GUIANA, GUIANA FRANCESA, SURINAME.

\section{N. unifascia Hebard, 1926}

unifascia Hebard, 1926:166; Bruijning, 1959:62; Rocha e Silva-Albuquerque \& Lopes, 1976:894.

Localidade-tipo: Macho. GUIANA FRANCESA (Nouveau Chantier), (ANSP). Distribuição geográfica: BRASIL (Amazonas), GUIANA FRANCESA.

\section{Descrição das genitálias das espécies de Neoblattella Shelford, 1911}

\section{N. adspersicollis}

(Figs. 4, 10, 16, 24, 30, 42 e 55)

Macho: a modificação tergal na região mediana apresenta-se no sétimo segmento em forma de cílios esparsos e no oitavo com cílios concentrados (Fig. 4). Placa supra-anal ciliada e pronunciada na região mediana (Fig. 10). Placa subgenital simétrica com estilos desenvolvidos e pouco afilados (Fig. 16).

Genitália: R2 em forma de gancho apicalmente em forma de unha (Fig. 42). L2vm bifurcado; L2d com espinhos pequenos e um espinho maior; ramo lateral do L2vm apicalmente arredondado com espinhos (Fig. 24). $\mathrm{L} 1 \mathrm{em}$ forma de $\mathrm{U}$ invertido com uma das extremidades maior e estrutura mediana com uma das bordas com espinhos (Fig. 30).

Material examinado: BRASIL: Amapá, Serra do Navio (1 macho e 1 fêmea), 9.I.1957; K. Lenko col.; Pará: Belém, I.A.N (1 macho e 1 fêmea), 10.IV.1963, Werner col.; localidade e coletor iguais, (1 macho), 4.IV.1963; Boa Vista, (1 macho), I.1965, W. França col.; Peixe-Boi, (1 macho e 1 fêmea), I.1964; Arlé col. (MNRJ). 


\section{N. binodosa}

(Figs. 17, 23, 31, 40, 41, 46, 50, 54 e 55)

Macho: a placa subgenital é simétrica com cílios lateralmente; estilos pequenos e alargados próximos à região centro-apical da placa (Fig. 17). A modificação tergal no abdome e a placa supra-anal não foram observadas. Genitália: R2 em forma de gancho robusto apicalmente terminando em forma de unha (Fig. 40). R3 em forma laminar, basalmente espatulado e apicalmente afilado (Fig. 41). L1 em forma de U invertido (Fig. 31). L2vm bifurcado, com L2d em forma de garra afilada e o ramo lateral com extremidade espatulada com várias proeminências pontiagudas (Fig. 23).

Fêmea: a placa supra-anal é curta e estreita, ciliada apicalmente e com reentrância mediana proeminente; cercos desenvolvidos e ciliados (Fig. 46). Placa subgenital oblonga com cílios nos bordos apicais (Fig. 50).

Genitália: válvulas desenvolvidas sendo o primeiro par mais robusto que os demais; segundo par muito afilado e subigual em tamanho ao primeiro par e o terceiro par muito desenvolvido em relação aos demais. Valvíferos laminares. Paratergitos desenvolvidos (Fig. 54).

Material examinado: BRASIL: Bahia*, Itabuna, (1 macho e 2 fêmeas), V.1971, Winder col. (em chão de cacau); Pará, Apeú, Castanhal, (1 macho), Il.1964, Arlé col.; Belém, Utinga, X.1965, P. Waldir col.; mesma localidade (1 fêmea), II.1965, R. Arlé col.; Belém, I.A.N., (1 macho), X.1965. W. França col.; Serra Tumucumaque, Tirios, (1 macho), II.1963, A. Machado col.; São Paulo, llha de São Sebastião, (1 macho), 02.IX.1957; Helga Urban col. (MNRJ).

\section{N. carvalhoi}

(Figs. 9, 15, 22, 29, 39 e 55)

Macho: a modificação tergal no abdome não foi observada. Placa supraanal é estreita com leve reentrância mediana (Fig. 9). Placa subgenital é simétrica com estilos pouco longos, próximos aos bordos da placa (Fig. 15). Genitália: L2vm bifurcado com L2d acuminado e o ramo lateral com proeminências pontiagudas (Fig. 22). L1 em forma de $U$ invertido com estrutura mediana apresentando espinhos nos bordos apicais (Fig. 29). R2 em forma de gancho distalmente em forma de unha (Fig. 39). 
Material examinado: BRASIL, Rio de Janeiro, Parque Nacional de Itatiaia, (Holótipo macho), 20.XI.1969, Santos \& Machado cols. (MNRJ).

\section{N. guianae}

(Figs. 1, 6, 12, 19, 26, 33, 34, 43, 47, 51 e 55)

Macho: a modificação tergal no sétimo segmento abdominal apresenta-se em forma de cílios esparsos e no oitavo segmento apresenta-se medianamente modificado (Fig. 1) Placa supra-anal proeminente medianamente com cílios esparsos; cercos muito desenvolvidos e afilados (Fig. 6). Placa subgenital simétrica com cílios nos bordos; estilos afilados (Fig. 12).

Genitália: R2 em forma de gancho terminado em uma unha apicalmente (Fig. 33). R3 em forma laminar afilada (Fig. 34). L1 como na figura 25. L2vm bifurcado, L2d com extremidade desenvolvida com espinhos e cílios no ápice; ramo lateral com extremidade menor levemente arredondada (Fig. 19).

Fêmea: a placa supra-anal apresenta-se com reentrância aprofundada na região mediana e apicalmente em forma de estruturas afiladas (Fig. 43). Placa subgenital é transversal e ciliada (Fig. 47).

Genitália: válvulas configuram-se em 3 pares semelhantes em forma e tamanho. Valvíferos afilados (Fig. 51).

Material examinado: BRASIL: Amapá, Sant. Ana, (1 fêmea), 18.IX.1957, L. Lane col.; Mato Grosso*, Sinop, (7 machos e 12 fêmeas), X.1974, Roppa \& Alvarenga cols.; mesma localidade e coletores (7 fêmeas), X/1975; Pará, Belém, I. A. N., (1 fêmea), X.1965, W. França col.; São Paulo*, Caraguatatuba (Res. Flor. 40m), (1 macho), 12.V a 1.VI.1962, Exp. Dep. Zool.col.; PERU, Ucayali, Pucallpa, Rio Ucuyali, (1 macho), III.1948, J. M. Schunke col., (MNRJ).

\section{N. longior}

(Figs. 2, 7, 13, 20, 27, 35, 36, 44, 48, 52 e 55)

Macho: a modificação tergal apresenta-se no sétimo segmento em forma de cílios esparsos (Fig. 2). Placa supra-anal estreita com bordo mediano proeminente com cílios esparsos; parapróctos visíveis e ciliados (Fig. 7). 
Placa subgenital é simétrica com cílios esparsos; estilos desenvolvidos próximos aos bordos da placa (Fig. 13).

Genitália: R2 em forma de gancho com ápice terminando em forma de unha (Fig. 35). R3 em forma laminar (Fig. 36). L1 em forma de U invertido, uma das extremidades mais desenvolvida, e estrutura mediana com o bordo de espinhos bastante esclerotinizado (Fig. 27). L2vm bifurcado. L2d apresentando apicalmente espinhos; ramo lateral com ápice em forma arredondado (Fig. 20).

Fêmea: a placa supra-anal apresenta-se com reentrância acentuada medianamente com os bordos ciliados; cercos afilados, ciliados e muito desenvolvidos (Fig. 44). Placa subgenital é oblonga com cílios esparsos (Fig. 48). Genitália: Segundo par de válvulas é afilado e reduzido; terceiro par muito desenvolvido e voltado para o interior apicalmente e o primeiro par mais alargado e maior que o segundo. Valvíferos reduzidos. Paratergitos muito desenvolvidos (Fig. 52).

Material examinado: BRASIL: Mato Grosso*, Vera, (5 fêmeas), X.1972, Alvarenga \& Roppa cols.; mesma localidade e coletores (1 macho e 4 fêmeas), X.1973; Pará, Iguapé-Assu, (1 macho), IX.1964, Souza \& Waldir cols.; Pará, Belém, I.A.N., (1 macho), X.1965, W. França col.; Belém, Utinga, (1 macho e 2 fêmeas), II.1965, Herbert col. (MNRJ).

\section{N. poecilops}

(Figs. 3, 8, 14, 21, 28, 37, 38, 45, 49, 53 e 55)

Macho: a modificação tergal apresenta-se no sétimo segmento abdominal com cílios esparsos no ápice (Fig. 3). Placa supra-anal é proeminente medianamente, e com cílios esparsos no ápice; cercos afilados e muito desenvolvidos (Fig. 8). Placa subgenital é simétrica, ciliada nos bordos com estilos pouco desenvolvidos localizados lateralmente no ápice da placa (Fig. 14).

Genitália: R2 em forma de gancho com extremidade distal em forma de unha (Fig. 37). R3 em forma laminar, simples e afilado (Fig. 38). L1 em forma de $\mathrm{U}$ invertido, uma das extremidades mais desenvolvida e uma estrutura mediana com espinhos esclerotinizados no bordo apical (Fig. 28). L2vm bifurcado próximo à base. L2d com extremidade mais desenvolvida com espinhos esclerotinizados; ramo lateral menor e de ápice arredondado (Fig. 21). 
Fêmea: a placa supra-anal apresenta o bordo apical ciliado com uma reentrância aprofundada medianamente; cercos ciliados e afilados apicalmente (Fig. 45). Placa subgenital é oblonga com cílios no bordo apical. (Fig. 49).

Genitália: válvulas apresentam-se com o primeiro par maior que os demais e o segundo e terceiro pares subiguais em forma e tamanho. Valvíferos são lamelares. Paratergitos são desenvolvidos (Fig. 53).

Material examinado: BRASIL: Mato Grosso, Vera, (2 machos e 2 fêmeas), X.1973, Alvarenga \& Roppa cols.; Pará, Belém, Utinga, (1 fêmea), I.1965, Herbert col.; Boa Vista, (1 fêmea), I.1965, W. França col.; I.A.N., (1 macho), IV.1963, Werner col.; Jambuaçu, Mojú, (1 macho), VII.1967, E. P. A. Z. \& M. G. cols.; Peixe-boi, (1 fêmea), 20.Il.1964, R. Arlé col.; Pirelli, (1 macho), VII.1965, Waldir col.; São Miguel da Guama, (1 macho), III.1964, Souza col.; Pernambuco*, Caruaru, Faz. Caruaru, 900 m.alt., (1 macho), IV.1972, M. Alvarenga col. (MNRJ).

\section{N. unifascia}

(Figs. 5, 11, 18, 25, 32 e 55)

Macho: a modificação tergal apresenta-se com cílios esparsos no sétimo segmento abdominal (fig. 5). Placa supra-anal é ampla, ciliada no bordo apical com leve reentrância medianamente; cercos desenvolvidos e ciliados (Fig. 11). Placa subgenital é simétrica, ciliado, com estilos medianamente desenvolvidos (Fig. 18).

Genitália: L2vm bifurcado basalmente. L2d com extremidade denteada; ramo lateral com ápice em forma de garra (Fig. 25). L1 em forma de U invertido contendo medianamente uma estrutura com bordo esclerotinizado (Fig. 32).

Material examinado: BRASIL, Amazonas, Manaus, (3 machos, 2 fêmeas e 13 ninfas), X.1974, (MNRJ).

\section{Chave das Espécies de Neoblattella Shelford, 1911 (com base em caracteres da genitália masculina)}

1. Placa subgenital simétrica com estilos alargados ............................. 2

1'.Placa subgenital simétrica com estilos afilados e desenvolvidos ........ 3 
2. Abdome com modificação mediana no sétimo tergito evidenciada por um grupo de cílios simétricos (Fig. 4). L2vm bífido. L2d com espinhos pequenos e um espinho maior. Ramo lateral do L2vm apicalmente arredondado com espinhos (Fig. 24). L1 com estrutura mediana alargada com espinhos (Fig. 30)

N. adspersicolis 2'. Abdome com modificação mediana no sétimo tergito com cílios esparsos. L2vm bífido. L2d em forma de garra afilada e o ramo lateral com extremidade espatulada com várias proeminências pontiagudas (Fig. 23). L1 com estrutura mediana linguiforme com estrutura esclerotinizada (Fig. 31)

N. binodosa

3. Placa supra-anal, entre os cercos, muito proeminente e estreita (Fig. 8) N. poecilops 3'. Placa supra-anal, entre os cercos, muito proeminente alargada (Fig. 11) N. unifascia 3".Placa supra-anal pouco proeminente 4

4. Abdome com modificação mediana no sétimo tergito com cílios esparsos e pequena diferenciação mediana no oitavo tergito (Fig. 1) ....... N. guianae 4'. Abdome com modificação mediana no sétimo tergito com cílios esparsos 5

5. L2vm bífido. L2d com extremidade desenvolvida com espinhos e cílios no ápice; ramo lateral com extremidade menor levemente arredondada (Fig. 20) N. longior 5'. L2vm bífido. L2d acuminado e ramo lateral com proeminências pontiagudas (Fig. 22)

N. carvalhoi

\section{Discussão}

Com base nas estruturas das peças genitais masculinas e das válvulas femininas, foi possível criar quatro grupos para englobar as espécies aqui estudadas:

a) Complexo I ou complexo longior (N. guianae, N. longior, N. poecilops, N. elegantula). Caracteriza-se por apresentar no macho o L2d com espinhos e ápice do ramo lateral arredondado. 
Distribuição Geográfica das Espécies de Neoblattella Shelford, 1911 (Fig. 55).

\begin{tabular}{|c|c|c|c|c|}
\hline & \multicolumn{3}{|c|}{ AMERICA DO SUL } & \multirow{3}{*}{$\begin{array}{l}\text { AMÉRICA } \\
\text { CENTRAL }\end{array}$} \\
\hline & \multicolumn{2}{|c|}{ BRASIL } & \multirow[b]{2}{*}{ OUTROS } & \\
\hline & ESTADOS & REGIÖES & & \\
\hline adspersicollis & $\begin{array}{c}\mathrm{AP}, \mathrm{PA}, \mathrm{CE}, \mathrm{BA}, \\
\mathrm{MT}, \mathrm{RJ}\end{array}$ & $\begin{array}{c}\text { Norte, Nordeste, } \\
\text { Centro-Oeste, } \\
\text { Sudeste }\end{array}$ & $\begin{array}{l}\text { Guiana Francesa, } \\
\text { Colômbia, }\end{array}$ & $\begin{array}{l}\text { Trinidad, Porto } \\
\text { Rico, Jamaica }\end{array}$ \\
\hline binodosa & $\mathrm{PA}, \mathrm{BA}^{*}, \mathrm{SP}$ & $\begin{array}{c}\text { Norte, Nordeste, } \\
\text { Sudeste }\end{array}$ & $\begin{array}{l}\text { Guiana Francesa, } \\
\text { Suriname }\end{array}$ & - \\
\hline carvalhoi & RJ & Sudeste & - & - \\
\hline elegantula & AM & Norte & Venezuela & - \\
\hline guianae & $\begin{array}{l}\mathrm{AP}, \mathrm{PA}_{\mathrm{SP}^{*}} \mathrm{MT}^{*}, \\
\end{array}$ & $\begin{array}{c}\text { Norte, Centro-Oeste, } \\
\text { Sudeste }\end{array}$ & $\begin{array}{l}\text { Guiana, Guiana } \\
\text { Francesa, Peru }\end{array}$ & - \\
\hline longior & $\mathrm{PA}, \mathrm{MT}^{*}$ & Norte, Centro-Oeste & $\begin{array}{l}\text { Guiana Francesa, } \\
\text { Suriname }\end{array}$ & - \\
\hline paulista & SP & Sudeste & - & - \\
\hline picta & $A P, R J, E S$ & Norte, Sudeste & - & - \\
\hline poecilops & $\mathrm{PA}, \mathrm{PE}^{*}, \mathrm{MT}$ & $\begin{array}{l}\text { Norte, Nordeste, } \\
\text { Centro-Oeste }\end{array}$ & $\begin{array}{l}\text { Guiana Francesa, } \\
\text { Suriname }\end{array}$ & - \\
\hline puerilis & RS & Sul & Argentina & - \\
\hline sucina & AM, PA & Norte & - & - \\
\hline tapenague & - & - & Argentina & - \\
\hline titania & $\mathrm{AM}, \mathrm{PA}$ & Norte & $\begin{array}{l}\text { Guiana, Guiana } \\
\text { Francesa, } \\
\text { Suriname }\end{array}$ & - \\
\hline unifascia & AM & Norte & Guiana Francesa & - \\
\hline
\end{tabular}

Devido a grande semelhança entre as genitálias dos machos de $N$. guianae, $N$. longior, $N$. poecilops a distinção entre elas se evidencia pelas fêmeas quanto à configuração das válvulas: em $N$. guianae as válvulas são subiguais em comprimento (Fig. 51); em $N$. longior, o terceiro par de válvulas é muito maior que os demais e voltado para dentro e o primeiro par muito alargado (Fig. 52); e em N. poecilops, o primeiro par é maior que os demais, arredondado no ápice, sendo os demais muito afilados e subiguais em comprimento (Fig. 53).

$N$. elegantula foi considerada próxima a $N$. adspersicollis em Rocha e Silva-Albuquerque (1964b), porém com base na placa supra-anal do macho apresentada pela autora, neste trabalho a colocamos no grupo longior.

b) Complexo II ou complexo carvalhoi ( $N$. carvalhoi). Caracteriza-se por apresentar no macho o L2d acuminado e o ramo lateral espatulado na extremidade com proeminências pontiagudas (Fig. 22); o L1 com um grupo de espinhos concentrados na região mediana (Fig. 29). 
c) Complexo III ou complexo adspersicollis ( $N$. adspersicollis, $N$. binodosa, N. paulista. Caracteriza-se por apresentar no macho o L2vm comespinhos pequenos na extremidade distal; ápice do ramo lateral de forma variada, de palmiforme até espiniforme (Figs. 23 e 24); a modificação tergal do abdome, no sétimo segmento, em forma de espiral (Fig. 4); o L1 alargado na região mediana com pequeno grupo de espinhos concentrados ou desprovido deles (Figs. 30 e 31).

Concordamos com Rocha e Silva-Albuquerque \& Gurney, 1963 quanto à $N$. paulista que foi colocada no grupo por ter sido considerada similar à $N$. binodosa e também em relação à $N$. puerilis já enquadrada por Hebard (1922) no grupo adspersicollis.

d) Complexo IV ou complexo unifascia (N. unifascia). Caracteriza-se por apresentar no macho a placa subgenital com estilos longos e afilados (Fig. 18); L2vm apicalmente com espinhos pequenos; ápice do ramo lateral afilado e espiniforme (Fig. 25); e L1 com esclerito longo e muito afilado (Fig. 32).

\section{Agradecimentos}

À Dra. Janira Martins Costa do Museu Nacional, Departamento de Entomologia, pelo apoio técnico.

\section{Referências Bibliográficas}

Becker, C.J.,1979. Neoblattella puerilis (Rehn, 1915) Epilampridae. Dictyoptera: nova ocorrência e descrição da fêmea. Rev. Bras. Entomol 23:19-21.

Bonfils, J.,1969. Contribution a l'étude des Blattidae des Antilles françaises, description d'espécies nouvelles (Dictyoptera-Blattaria). Ann. Soc. Entomol. Fr. 5:109-135.

Bruijning, C.F.A., 1959. The Blattidae of Surinam. Studies on the Fauna of Suriname and other. Stud. Fauna Suri. 2:1-103. 
Bruner, L., 1906. Report on the Orthoptera of Trinidad, West Indies. J. N. Y. Entomol. Soc. 14:135-165.

Brunner, von, C. W., 1865. Nouveau système des Blattaires. Societè. I.R. Zoologie et de Botanique., 426 p.

Brunner, von, C. W. \& Redtenbacher, J., 1892. On the Orthoptera of the Island of St. Vincent, West Indies. Proc. Zool. Soc. 1892:196-220.

Brunner, von, C.W., 1893. On the Orthoptera of the Island of Grenada, West Indies. Proc. Zool. Soc. 1893:599-611.

Caudell, A.N., 1913. Description of two new species of Orthoptera from Peru. Can. Entomol. 45:19-21.

Gurney, A.B., 1942. Studies in Cuba Blattidae (Orthoptera). Bull. Mus. Comp. Zool. 89:11-60.

Gowdey, C. C.,1926. Catalogue insectorum jamaicensis. Dep. Agric. Jamaica. Entomol. Bull. 4:1-11.

Hebard, M.,1916. A new species of the genus Neoblattella from Costa Rica. Entomol. News 27:159-161.

Hebard, M.,1917. The Blattidae of North American North of the Mexican Boundary. Mem. Am. Entomol. Soc. 2:1-284.

Hebard, M., 1919. The Blattidae of Panama. Mem. Am. Entomol Soc. 4:1-148.

Hebard, M., 1922. South American Blattidae from the Museum National d'Histoire Naturelle. Paris, France. Proc. Acad. Nat. Sci. 73:193-304.

Hebard, M.,1926. The Blattidae of French Guiana. Proc. Acad. Nat. Sci. 78:135-244.

Hebard, M.,1929. Previously unreported Tropical American Blattidae (Orthoptera) in the British Museum. Trans. Am. Entomol. Soc. 55:345-488. 
Hebard, M.,1932. New Species and records of Mexican Orthoptera. Trans. Am. Entomol. Soc. 58:201-371.

Kirby, W.F., 1904. A synonymic catalogue of Orthoptera. Euplexoptera, Cursoria (Forficulidae, Hemimeridae, Blattidae, Mantidae, Phasmidae). Volume 1. Ed. British Museum, London. 501 p.

Lopes, S.M. \& Oliveira, E.H. de, 2000. Espécie Nova de Eublaberus Hebard, 1919 do Estado de Goiás, Brasil e notas sobre E. marajoara Rocha e Silva Albuquerque,1972 (Blaberidae, Blaberinae). Bol. Mus. Nac. 433:1-5.

Princis, K. \& Kevan, D. K., 1955. Cockroaches (Blattariae) from Trinidad, B. W. J., with a few records from other parts of the Caribbean. Opusc. Entomol. 20:149-169.

Rehn, J.A.G., 1903. Studies in American Blattidae. Trans. Am. Entomol. Soc. 29:259-290.

Rehn, J.A.G., 1915. A further contribution to the knowledge of the Orthoptera of Argentina. Proc. Acad. Nat. Sci. 67:270-292.

Rehn, J.A.G., 1916. The Stanford expedition to Brazil, 1911, J. C. Branner, Director. Dermaptera and Orthoptera. Trans. Am. Entomol. Soc. 42:215-308.

Rehn, J.A.G., 1932. Wissenschaftliche Ergebnisse der Schwedischen entomologischen Reisen des Herrn Dr A. Roman in Amazonas 19141915 und 1923-1924. Arkiv. Zool. 24A:1-73.

Rehn, J. A.G., 1949. Cataloque of the Orthoptera of Venezuela. I. Blattidae. Bol. Entomol. Venez. 8:11-19.

Rehn, J.A.G. \& Hebard, M., 1905. A contribution to the knowledge of the Orthoptera of South and Central Florida. Proc. Acad. Nat. Sci. 57:20-52.

Rehn, J. A. G. \& Hebard, M., 1912. On Orthoptera found on the Florida keys and in extreme Southern Florida I. Proc. Acad. Nat. Sci. 54:235-276. 
Rehn, J.A.G. \& Hebard, M., 1914. On Orthoptera found on the Florida Keys and in extreme Southern Florida II. Proc. Acad. Nat. Sci. 56:373-412.

Rehn, J.A.G. \& Hebard, M., 1927. The Orthoptera of the West Indies. Number I. Blattidae. Bull. Am. Mus. Nat. Hist. 54:320.

Rocha e Silva-Albuquerque, I., 1964a. Check-List dos Blattaria Brasileiros. Bol. Mus. para. Emilio Goeldi, 41:1-37.

Rocha e Silva-Albuquerque, I., 1964b. On Collection of Cochroaches from Venezuela (Orthoptera, Blattoidea). Bol. Mus. para. Emilio Goeldi, 45:1-22.

Rocha e Silva-Albuquerque, I., 1964c. Novas Ocorrências de Blattellinae no Peru com descrição de três espécies novas (Epilampridae). Bol. Mus. para. Emilio Goeldi, 50:1-8.

Rocha e Silva-Albuquerque, I., 1965. Novas Ocorrências de Blattaria no Brasil e descrição de três espécies novas (Orth. Blattoidea). Bol. Mus. Nac. 57:1-11.

Rocha e Silva-Albuquerque, I., 1972. Inventário dos Blattaria da Amazônia com descrição de três espécies nova. Bol. Mus. para. Emilio Goeldi, 76:1-38.

Rocha e Silva-Albuquerque, I. \& Gurney, A.B., 1962. Insecta Amapaensia. Orthoptera: Blattodea. Stud. Entomol. 5:235-256.

Rocha e Silva-Albuquerque, I. \& Gurney, A.B.,1963. Records and descriptions of cockroaches from Southern Brazil (Orthoptera, Blattoidea). Stud. Entomol. 6:515-536.

Rocha e Silva-Albuquerque, I. \& Lopes, S. M., 1976. Blattaria de Bromélia (Dictyoptera). Rev. Bras. Biol. 36:873-901.

Rocha e Silva, I. 1982. Lista dos Blattodea do Município do Rio de Janeiro, RJ, BRASIL (Dictyoptera). Bol. Mus. Nac. 304:1-20. 
Saussure, H. de, 1870. Famille des Blattides . Volume 4:1-123. In: Recherches zoologique pour servir a l'histoire de la faune de l'Amérique Centrale et du Mexique. Imprimerie Impériale, Paris. 123 p.

Saussure, H. de \& Zehntner, L., 1893. Insecta, Orthoptera, Blattidae 1:13123. In: Biologia Centrali-Americana. Ed. British Museum, London. $123 \mathrm{p}$.

Shelford, R., 1908. Orthoptera. Fam. Blattidae. Subfam. Phyllodromiinae. Genera Insect. 73:1-29.

Shelford, R., 1911. Preliminary diagnoses of some new genera of Blattidae. Ent. Month. Mag. 47:154-156

Stal, C., 1858-1861. Orthoptera. Zool. Vol. V: 229-350. In: Konglia Evenska Fregatten Eugenies resa omkring jordens. Stockholm. 350 p.

Walker, F., 1868. Catalogue of Specimens of Blattariae in Collection of British Museum:239 p.

Wolcott, G.N., 1924. Insectae Portoricencis. A preliminary annotated check list of Insects of Porto Rico, with of some new species. J. Dept. Agric. 7:1-313. 


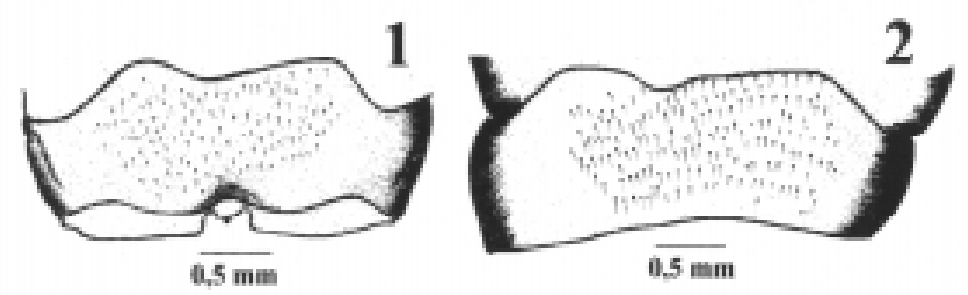

COMPLEXO I

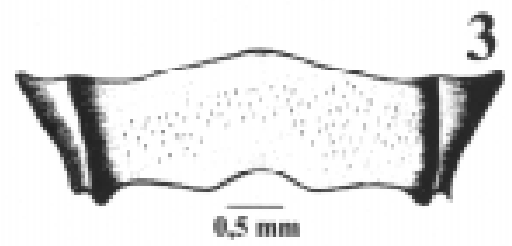

COMPLEXO III

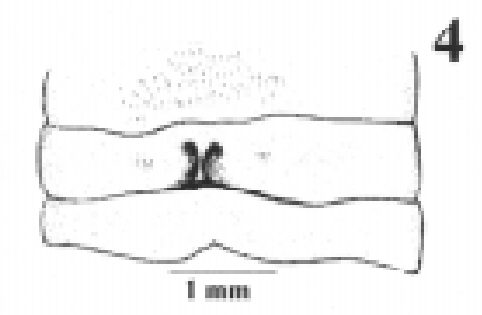

COMPLEXO IV

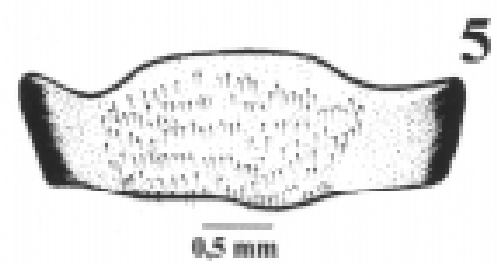

Modificação tergal (macho), vista dorsal. Complexo I - Fig. 1: N. guianae Hebard, 1929; Fig. 2: N. longior Hebard, 1926; Fig. 3: N. poecilops Hebard, 1926; Complexo III - Fig. 4: N. adspersicollis (Stal, 1858); Complexo IV - Fig. 5: N. unifascia Hebard, 1926. 

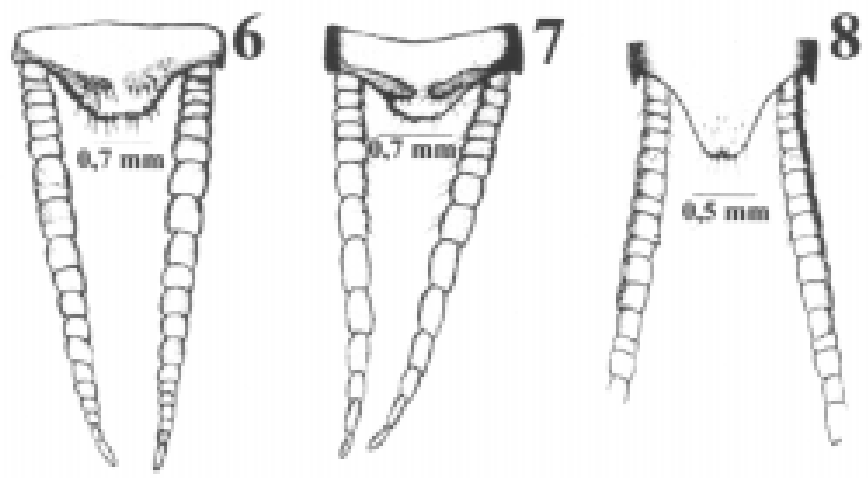

\section{COMPLEXO I}

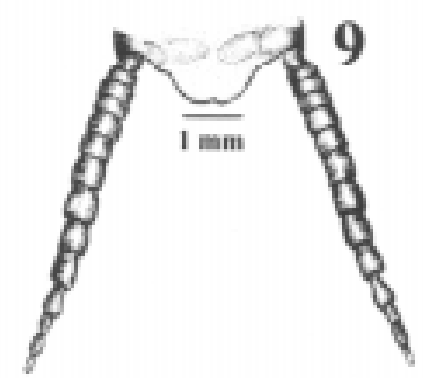

COMPLEXO II

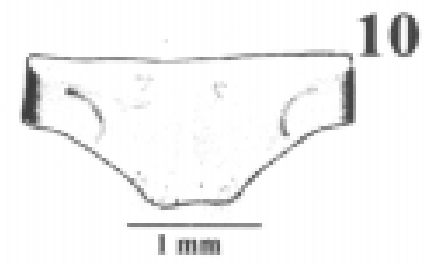

COMPLEXO III

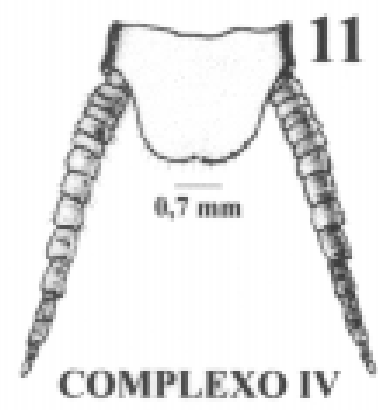

Placa Supra-anal (macho), vista dorsal Complexo I - Fig. 6: N. guianae Hebard, 1929; Fig. 7: N. longior Hebard, 1926; Fig. 8: N. poecilops Hebard, 1926; Complexo II - Fig. 9: N. carvalhoi Rocha e Silva-Albuquerque \& Lopes, 1976; Complexo III - Fig. 10: N. adspersicollis (Stal, 1858); Complexo IV - Fig. 11: $\mathbf{N}$. unifascia Hebard, 1926. 

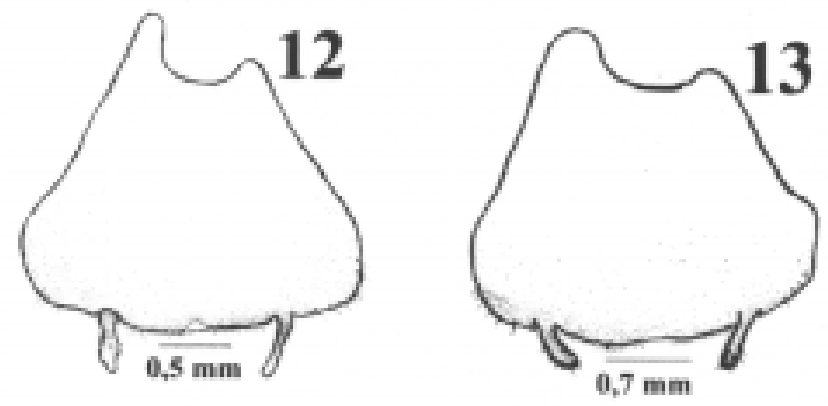

\section{COMPLEXO I}

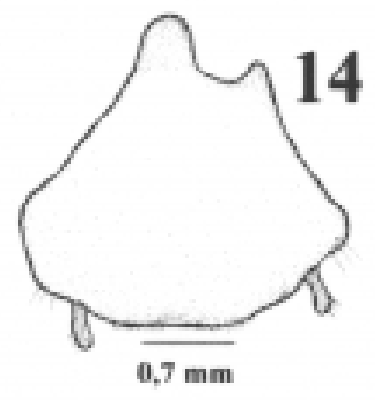

\section{COMPLEXO II}

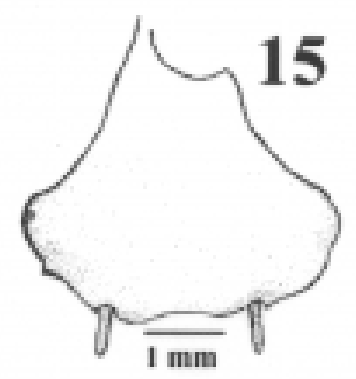

Placa Subgenital (macho), vista ventral. Complexo I - Fig. 12: N. guianae Hebard, 1929; Fig. 13: N. longior Hebard, 1926; Fig. 14: N. poecilops Hebard, 1926; Complexo II - Fig. 15: N. carvalhoi Rocha e Silva-Albuquerque \& Lopes, 1976. 


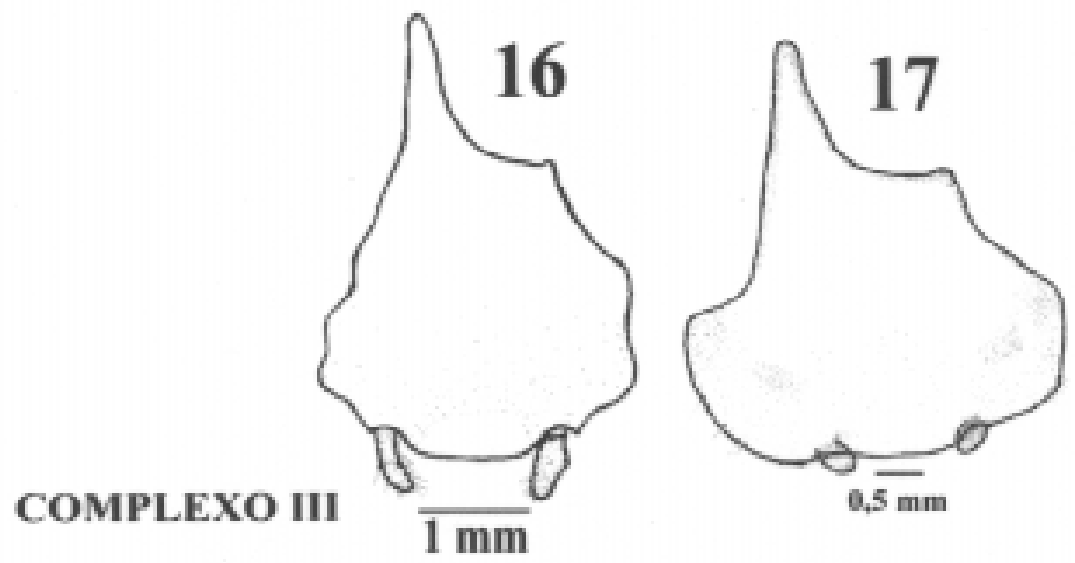

\section{COMPLEXO IV}

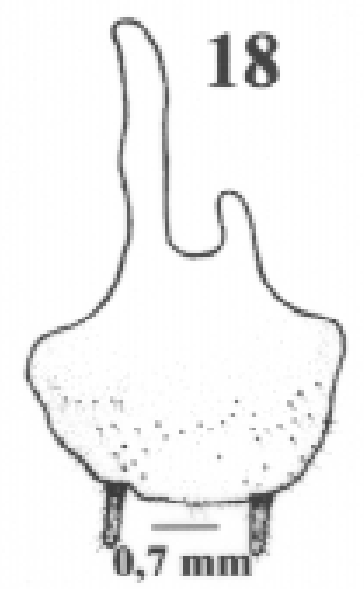

Placa Subgenital (macho), vista ventral. Complexo III - Fig. 16: $\mathbf{N}$. adspersicollis (Stal, 1858); Fig. 17: N. binodosa Hebard, 1926; Complexo IV Fig. 18: N. unifascia Hebard, 1926. 

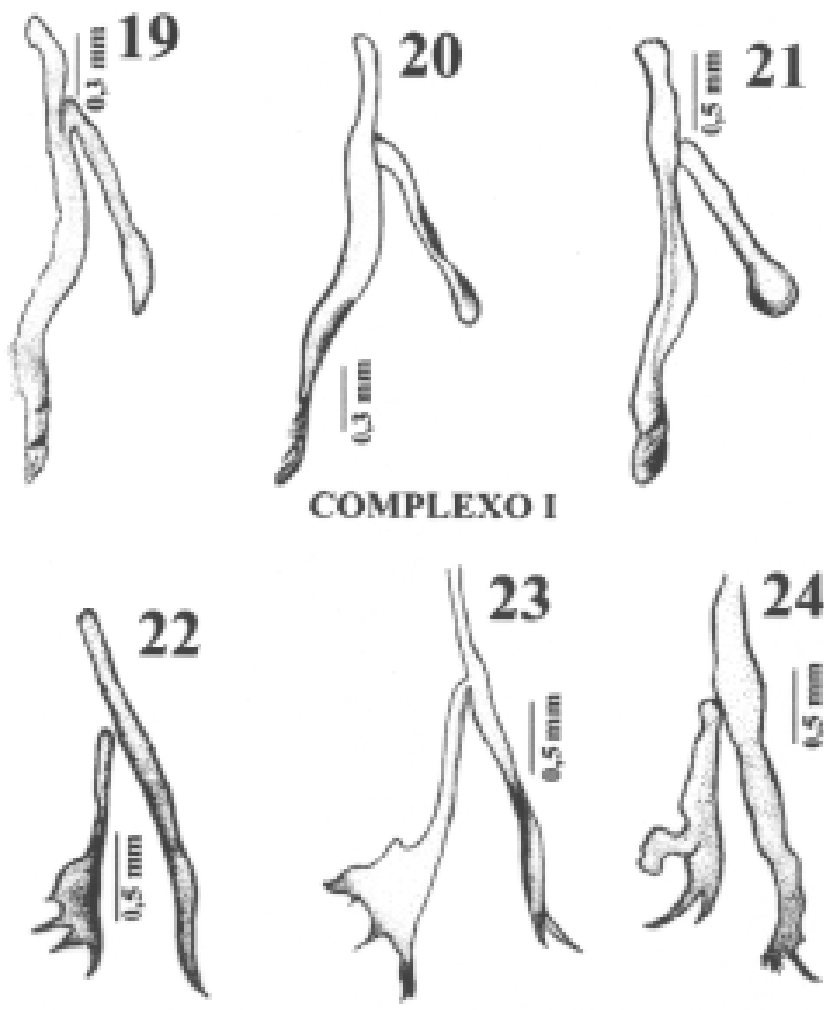

COMPLEXO I

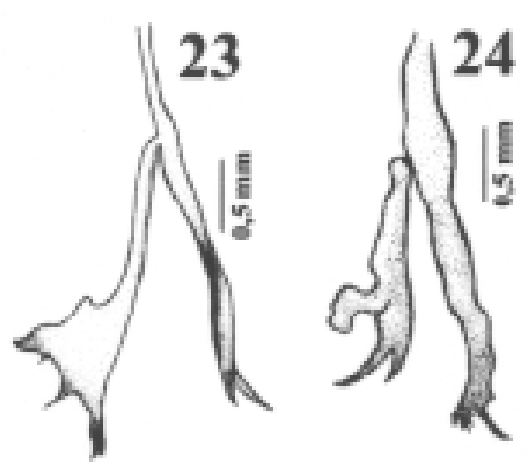

COMPLEXO II

\section{COMPLEXO III}

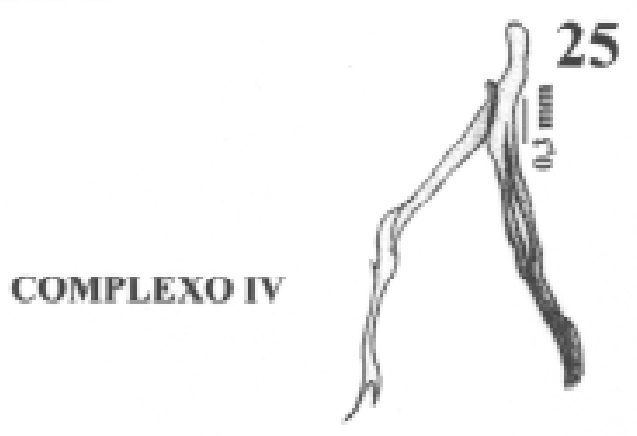

Esclerito mediano - L2vm (macho), vista dorsal. Complexo I - Fig. 19: $\mathbf{N}$. guianae Hebard, 1929; Fig. 20: N. longior Hebard, 1926; Fig. 21 : N. poecilops Hebard, 1926; Complexo II - Fig. 22: N. carvalhoi Rocha e Silva-Albuquerque \& Lopes, 1976; Complexo III - Fig. 23: $\mathbf{N}$. binodosa Hebard, 1926; Fig. 24: $\mathbf{N}$. adspersicollis (Stal, 1858); Complexo IV - Fig. 25: N. unifascia Hebard, 1926. 

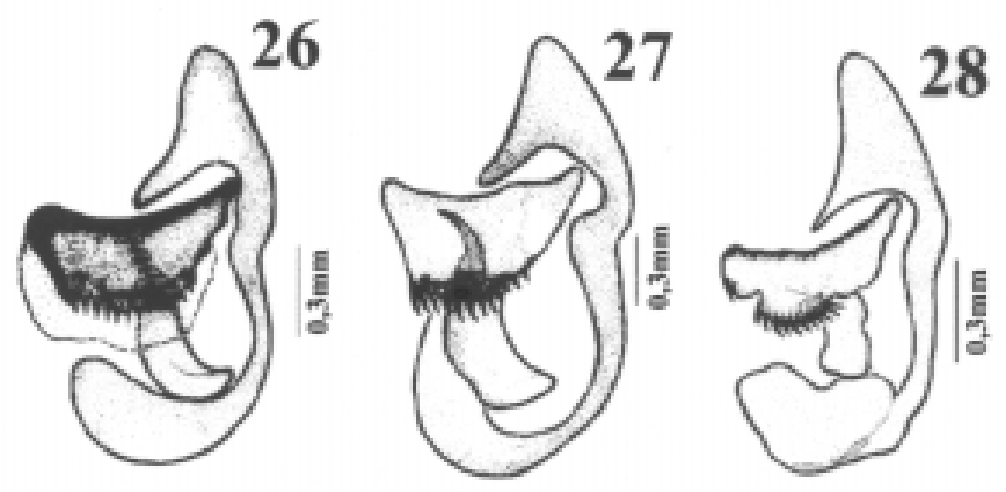

COMPLEXO I

COMPLEXO II

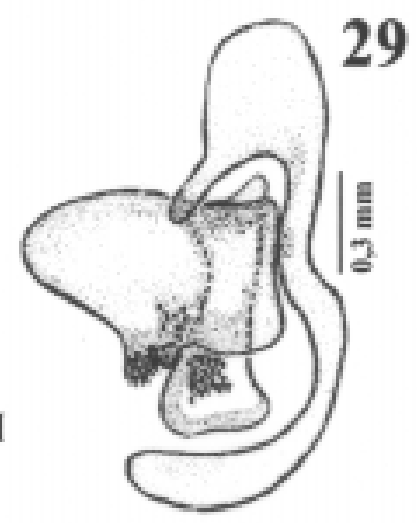

Falômero esquerdo - L1 (macho), vista dorsal. Complexo I - Fig. 26: $\mathbf{N}$. guianae Hebard, 1929; Fig. 27: N. longior Hebard, 1926; Fig. 28: N. poecilops Hebard, 1926; Complexo II - Fig. 29: N. carvalhoi Rocha e Silva-Albuquerque \& Lopes, 1976. 

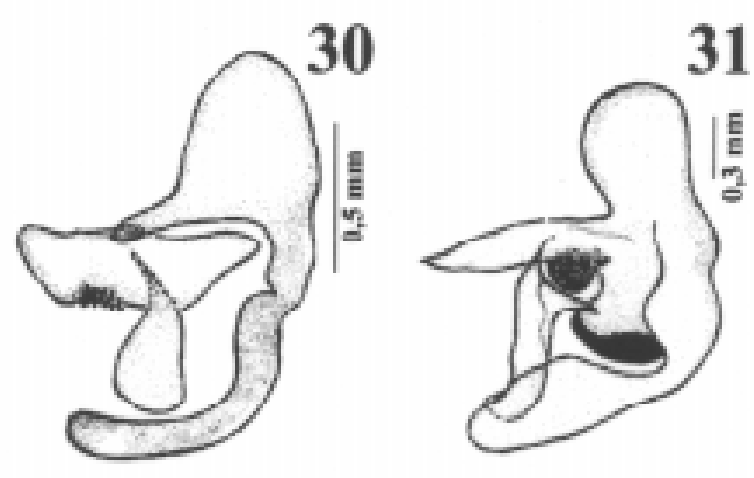

COMPLEXO III

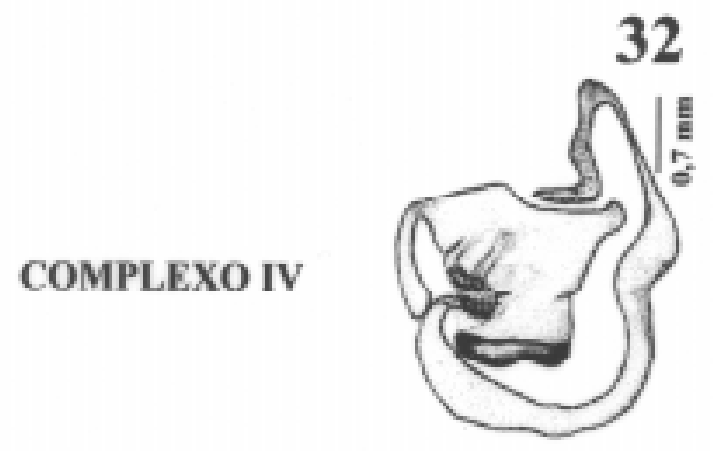

Falômero esquerdo - L1 (macho), vista dorsal. Complexo III - Fig. 30: N. adspersicollis (Stal, 1858); Fig. 31: N. binodosa Hebard, 1926; Complexo IV Fig. 32: N. unifascia Hebard, 1926. 

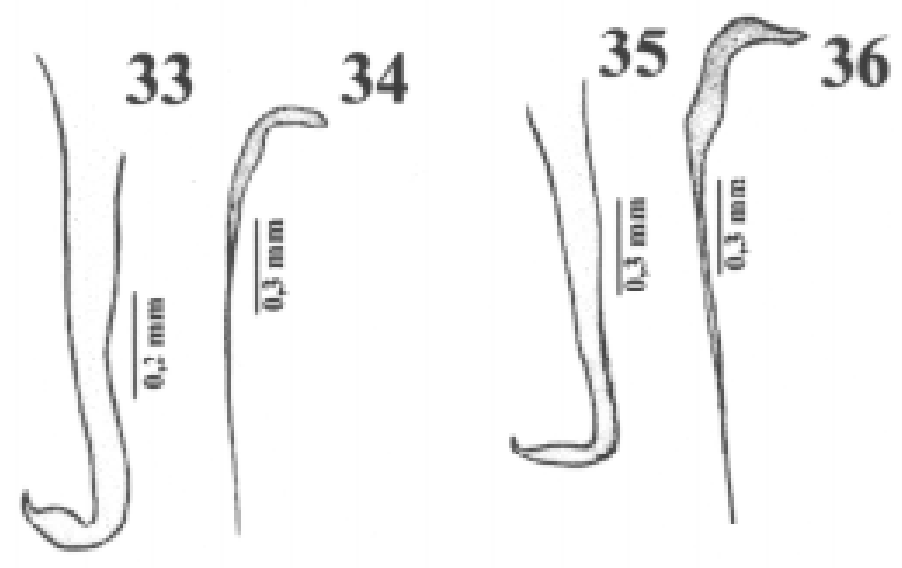

COMPLEXO I

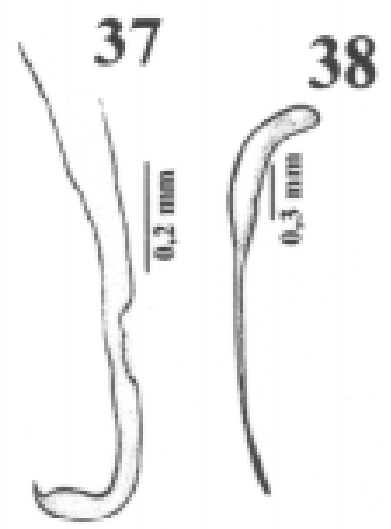

Falômero direito (R2) e esclerito (macho), vista dorsal. Complexo I - Figs. 33 e 34: N. guianae Hebard, 1929; Fgis. 35 e 36: N. longior Hebard, 1926; Figs. 37 e 38: N. poecilops Hebard, 1926. 


\section{COMPLEXO II}
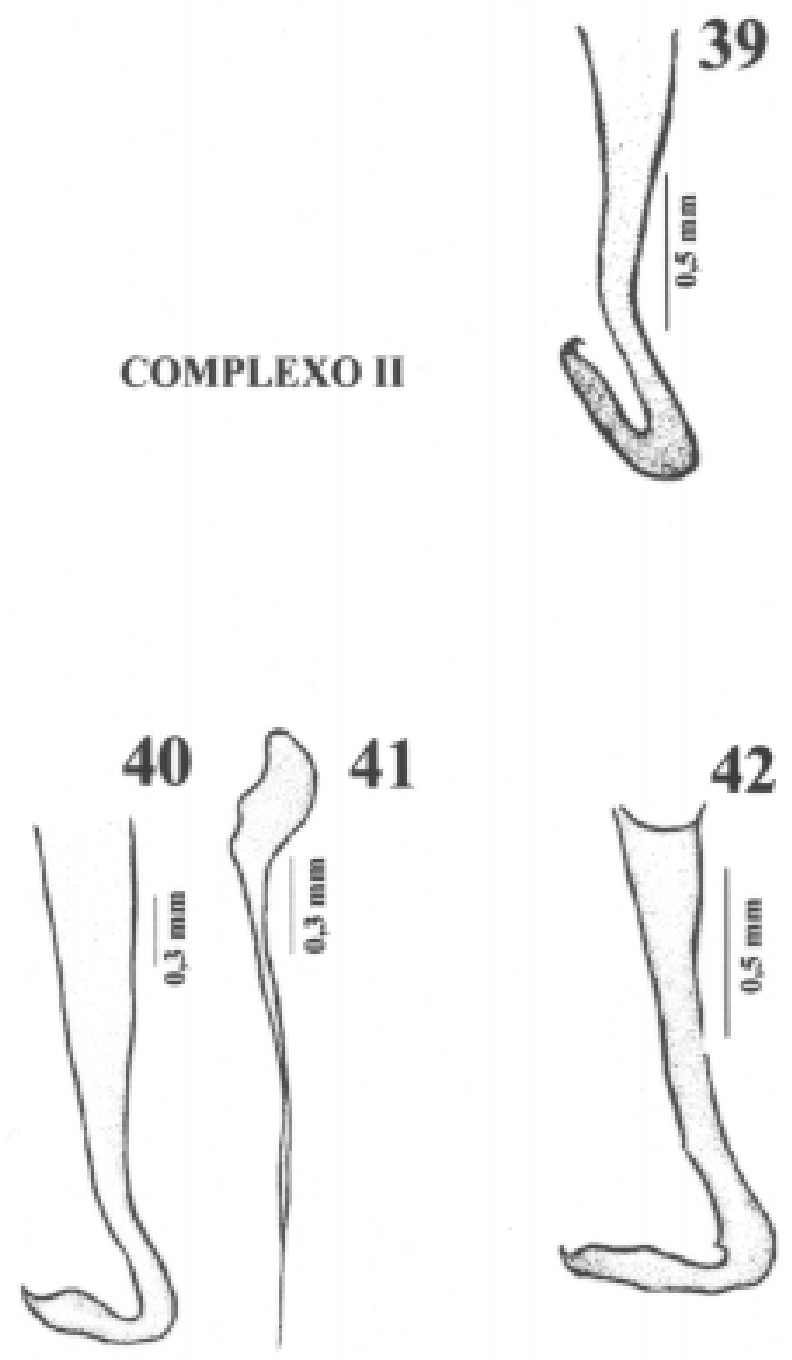

COMPLEXO III

Falômero direito (R2) e esclerito (macho), vista dorsal. Complexo II - Fig. 39: N. carvalhoi Rocha e Silva-Albuquerque \& Lopes, 1976; Complexo III Figs. 40 e 41: N. binodosa Hebard, 1926; Fig. 42: N. adspersicollis (Stal, 1858). 


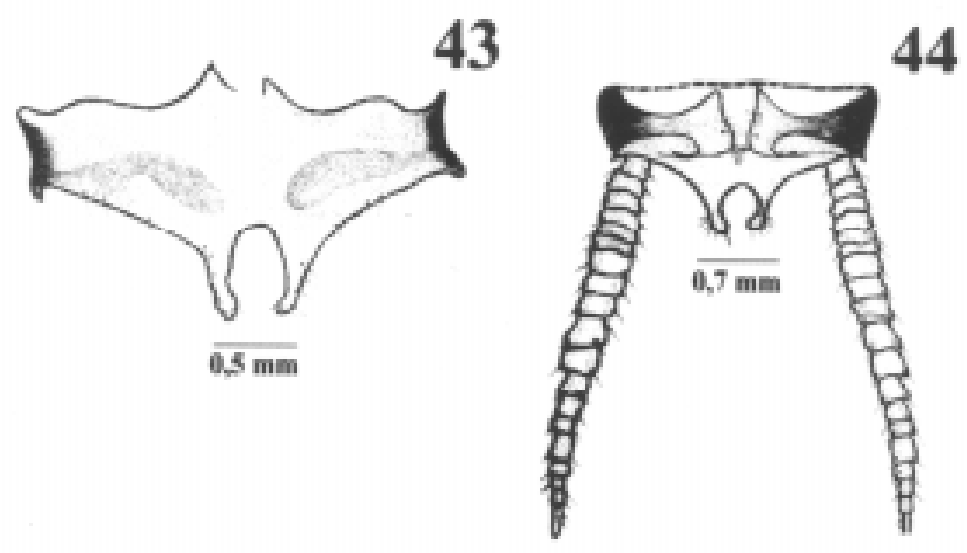

COMPLEXO I

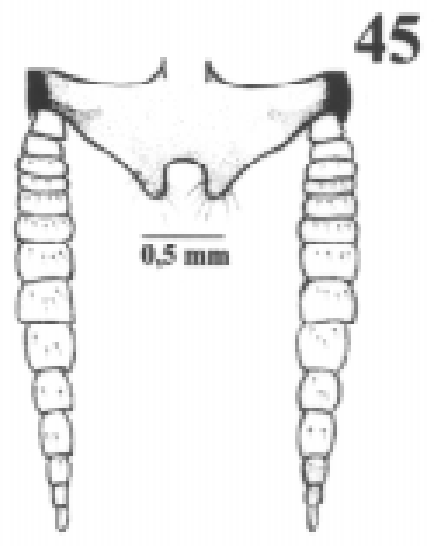

COMPLEXO III

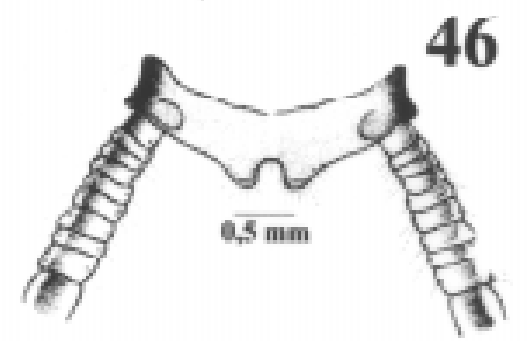

Placa supra-anal (fêmea), vista dorsal. Complexo I - Fig. 43: N. guianae Hebard, 1929; Fig. 44: N. longior Hebard, 1926; Fig. 45: N. poecilops Hebard, 1926; Complexo III - Fig. 46: N. binodosa Hebard, 1926. 

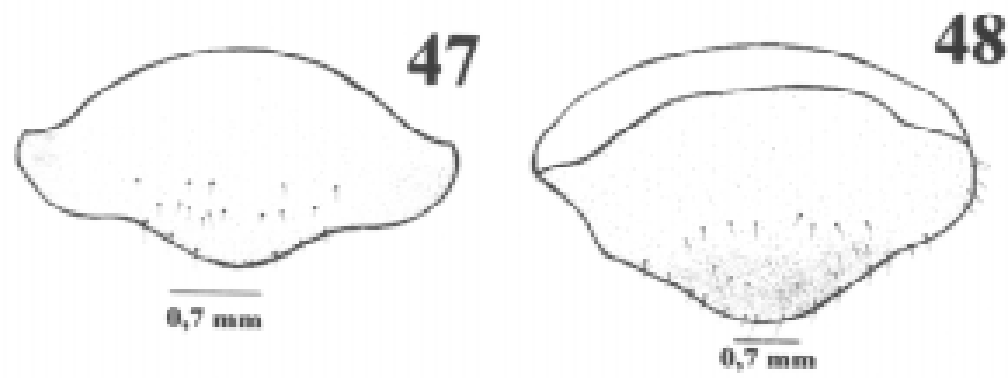

COMPLEXO I

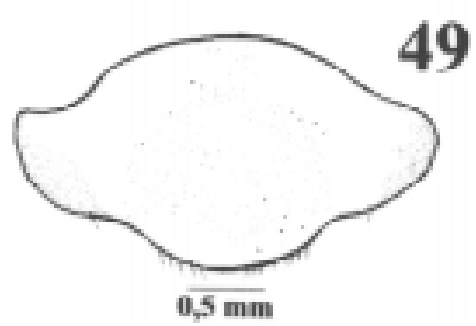

COMPLEXO III

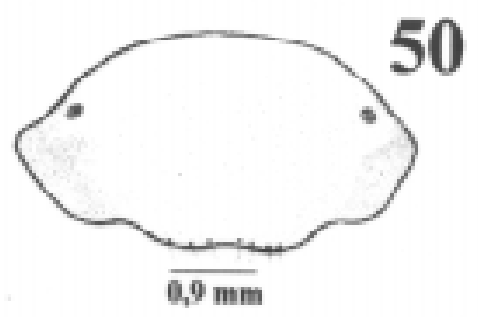

Placa Subgenital (fêmea), vista ventral. Complexo I - Fig. 47: N. guiane Hebard, 1929; Fig. 48: N. longior Hebard, 1926; Fig. 49: N. poecilops Hebard, 1926; Complexo III - Fig. 50: N. binodosa Hebard, 1926. 


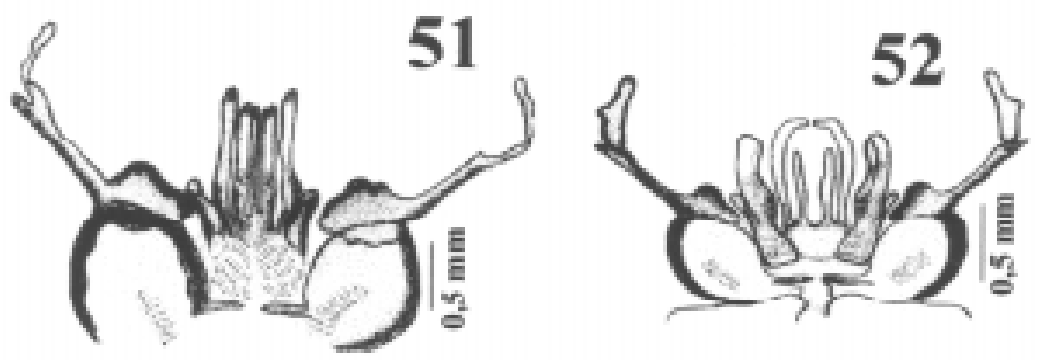

COMPLEXO I

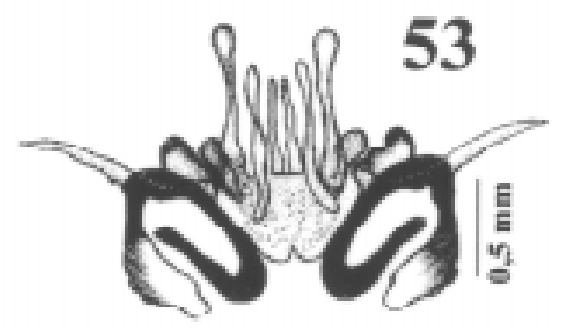

COMPLEXO III

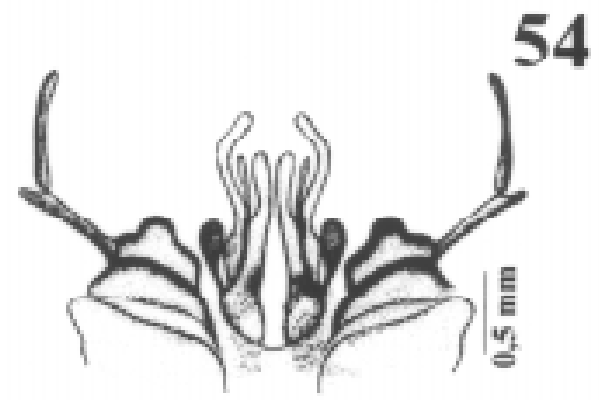

Válvulas (fêmea), vista dorsal. Complexo I - Fig. 51: N. guianae Hebard, 1929; Fig. 52: N. longior Hebard, 1926; Fig. 53: N. poecilops Hebard, 1926; Complexo III - Fig. 54: N. binodosa Hebard, 1926. 


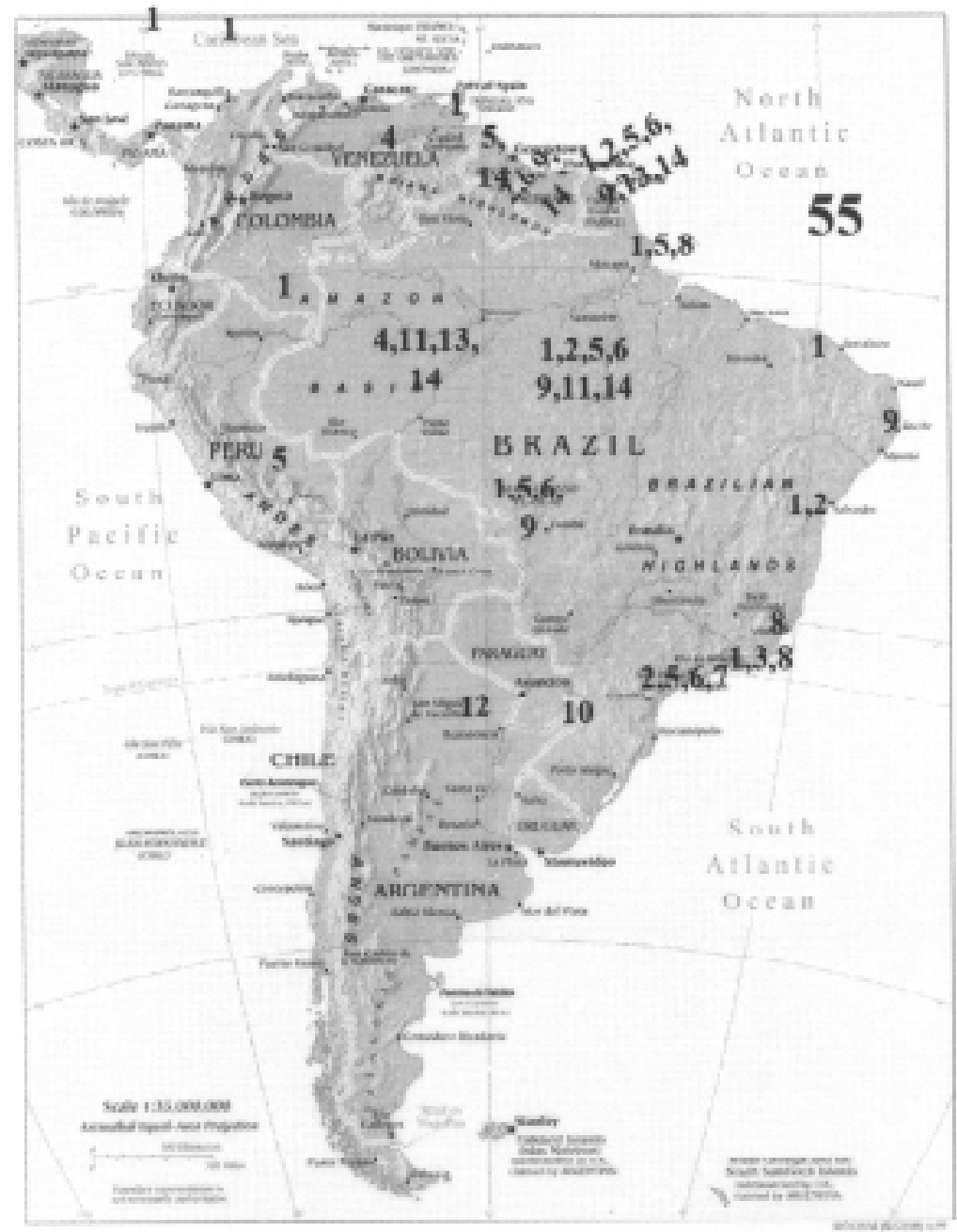

N. adspersicollis (Stal, 1858) (1)

N. picta R.S.-Albuquerque \& Gurney, 1962 (8)

N. binodosa Hebard, 1926 (2)

N. poecilops Hebard, 1926 (9)

N. carvalhoi R.S.-Albuquerque \& Lopes, 1976 (3)

N. puerilis Rehn, 1915 (10)

N. elegantula R.S.-Albuquerque, 1964 (4)
N. sucina Rehn, 1932 (11)

N. guianae Hebard, 1929 (5)

N. tapenagae Hebard, 1922 (12)

N. longior Hebard, 1926 (6)

N. unifascia Hebard, 1926 (13)

N. paulista R.S.-Albuquerque \& Gurney, 1963 (7)

N. titania (Rehn, 1903) (14)

Figura 55: Mapa da Distribuição Geográfica das Espécies Neotropicais de Neoblattella Shelfod, 1911. 